\title{
Personal Factors Influencing Patients' Adherence to ART in Addis Ababa, Ethiopia
}

\author{
Tefera Negash, MPH \\ Valerie Ehlers, D Litt et Phil
}

Our study attempted to identify personal (patientrelated) factors influencing antiretroviral therapy (ART) adherence in Addis Ababa, Ethiopia. A quantitative, descriptive design was used. Structured interviews were conducted with 355 HIV-infected patients on ART. The findings revealed that stigma, discrimination, depression, and alcohol use negatively affected patients' ART adherence levels. However, patients' knowledge levels had no influence on their ART adherence levels, contrary to other researchers' reports. Addressing stigma and discrimination at community levels might enhance patients' abilities to take their medications in the presence of others. Health care professionals should be educated to diagnose and treat depression in patients during the early stages of ART administration. Patients who are nonadherent to ART should be counseled about potential alcohol abuse. Stigma-related challenges also need to be addressed.

(Journal of the Association of Nurses in AIDS Care, 口, 1-9) Copyright (C) 2013 Association of Nurses in AIDS Care

Key words: adherence, antiretroviral, antiretroviral treatment, depression, discrimination, Ethiopia, HIV infection, stigma

A adherence rate of at least $95 \%$ is necessary to maximize the benefits of antiretroviral therapy (ART). These benefits include improved clinical, immune, and viral parameters (World Health Organization [WHO], 2006). An adherence rate of less than $95 \%$ is associated with higher levels of viral drug resistance, which has public health implications, and poor personal health outcomes (WHO, 2003).
Adherence can be measured using different techniques including medication event monitoring systems, pill counts, biologic markers, pharmacy refill data, and patient self-reports (WHO, 2003).

Patients with lower adherence levels have higher viral load levels, making them more likely to transmit HIV to sexual partners in comparison to those with lower viral load levels (Wutoh et al., 2001). Ultimately, it is the patient him/herself who does or does not adhere to the prescribed ART regimen, making an investigation into personal factors affecting ART adherence essential. A study conducted in the southern part of Ethiopia reported that the main personal reasons for nonadherence to ART included being busy, simply forgetting, changes in daily routine, and being away from home (Markos, Worku, \& Davey, 2008). A Zambian study identified patient-related factors that facilitated ART adherence, such as looking and feeling better, the support of the patient's family, physical reminders (such as watches or clocks) to take the prescribed drugs, and seeing ill people regain their health on ART. Rumors about ART and experiencing side effects, especially increased hunger, affected ART adherence levels negatively (Grant, Logie, Masura, Gorman, \& Murray, 2008).

Tefera Negash, MPH, is a Master of Public Health Graduate, Department of Health Studies, University of South Africa, Pretoria, South Africa. Valerie Ehlers, D Litt et Phil, is a Professor in the Department of Health Studies, University of South Africa, Pretoria, South Africa. 


\section{Key Concepts}

Antiretroviral medications (ARVs) are used in the treatment of HIV. ART requires the administration of at least three different ARVs in order to suppress the replication of HIV (WHO, 2006). The first-line ARV regimen for adults and adolescents contains two nucleoside reverse transcriptase inhibitors (NRTI) and one nonnucleoside reverse transcriptase inhibitor (NNRTI). For patients who develop treatment failure, the preferred second-line ARV regimen includes two NRTIs and one protease inhibitor (WHO, 2006). Successful ART adherence requires that the patient's behaviors in taking medications, following a diet, and/or executing lifestyle changes correspond with agreed-upon recommendations from a health care provider (WHO, 2003).

In our study, adherence was determined by a patient's behavior in taking ARVs as prescribed by a health care worker. It was assumed that persons living with HIV infection (PLWH) with ART adherence levels greater than or equal to $95 \%$ would have better health outcomes, improved immune responses, and suppressed viral loads. We assumed that optimal adherence, of at least 95\%, would be enhanced by knowledge about HIV and ART, minimal stigma and discrimination, absence of depression, and low levels of alcohol intake.

Depression was defined as a depressed mood on a daily basis for a minimum duration of 2 weeks. Depression also can manifest in the form of loss of interest or pleasure in nearly all activities, including activities usually enjoyed by the patient (Reus, 2005).

Discrimination refers to negative attitudes or particular behaviors or actions toward others. It is often described as a distinction made about persons resulting in unjust treatment because they belong to, or are perceived to belong to, a particular group (EngenderHealth, 2004). The operational definition of discrimination used in our study was unfair treatment of PLWH by their communities, family members, and/or health care providers, based on their HIV status.

Stigma is an undesirable or discrediting attribute that a person or group possesses that reduces personal or group status in society (EngenderHealth, 2004). The operational definition used in our study was a person's or a group's undesirable feelings toward PLWH.

\section{Purpose, Aims, and Objectives}

Different variables under patient-related factors were studied to identify their effects on adherence or nonadherence, including patients' knowledge about HIV and ART, HIV stigma and discrimination, depression, and alcohol use. Scaling up of the ART program throughout Ethiopia has resulted in increasing numbers of patients on ART (Ethiopian Federal Ministry of Health HIV/AIDS Prevention and Control Office [EFMOH/HAPCO], 2006). Our study proposed to identify patient-related factors that might influence ART adherence rates in Addis Ababa. The results could contribute to the body of knowledge of adherence in Ethiopia and provide recommendations and directions toward enhancing ART adherence levels. The objectives of the study were to:

1. Determine the ART adherence status of patients in Addis Ababa, Ethiopia.

2. Identify the influence of patients' knowledge of HIV and ART on their adherence levels in Addis Ababa, Ethiopia.

3. Assess the influence of HIV stigma and discrimination on ART adherence in Addis Ababa, Ethiopia.

4. Evaluate the influence of depression on ART adherence in Addis Ababa, Ethiopia.

5. Identify the influence of alcohol use on ART adherence in Addis Ababa, Ethiopia.

\section{Methods}

\section{Design}

A quantitative, descriptive, and cross-sectional study was conducted. Our study investigated the determinants of ART adherence and addressed some of the challenges affecting ART adherence in Addis Ababa.

\section{Research Site}

HIV prevalence in the overall Ethiopian population is $2.4 \%$; the urban prevalence is $7.7 \%$, while the rural 
prevalence is $0.9 \%$. Addis Ababa is the capital city of Ethiopia, and the adult prevalence rate of HIV in this area is $9.2 \%$ (EFMOH/HAPCO, 2007).

The research was conducted at one hospital because it provided treatment to a large number of patients on ART. Our study focused on the influences of personal factors on ART adherence levels. Thus, selecting an urban site that provided services to a large number of patients and that had adequate drug supplies was deemed suitable for identifying personal factors affecting ART levels, rather than challenges attributable to the health care system. A reported 4,708 patients received ART from this hospital by the end of February 2009 (EFMOH/HAPCO, 2009). The participating hospital was accessible to the data collectors, and the hospital management encouraged research about personal factors affecting patients' ART adherence levels. Most patients at the hospital could speak Amharic and/or English and would thus be able to communicate with the interviewers, who were conversant in both languages.

\section{Study Population and Sample}

According to Burns and Grove (2005), a target population is the entire set of individuals who meet the sampling (inclusion) criteria. In Ethiopia, most ART clinics are attached to, or operate in collaboration with, public hospitals. Only a small number of private hospitals and clinics provide ART services in a few large cities. The population for our study comprised patients on ART at one hospital's ART clinics in Addis Ababa. The inclusion criteria were: PLWH who were at least 18 years of age and who had been on ART for at least 12 months. The exclusion criteria were those patients on ART who were younger than 18 years of age or who had used ART for less than 12 months.

Patients on ART were chosen using simple random-sampling techniques. Potential respondents were identified using their ART unique numbers. These numbers were obtained from the ART clinic and randomly chosen using a table of random numbers until the desired sample size had been reached. Selected ART numbers were crosschecked with the patients' identifications and each selected patient was contacted when s/he came to the clinic for ARV refills. Selected patients were in- terviewed after they had voluntarily agreed to participate in the study and signed informed consent forms.

The formula used for the sample size calculation was $S=p(1-p) z^{2} / d^{2}$, where $p$ indicated the anticipated population proportion, $z$ referred to the cutoff value of the normal distribution, and $d$ was the precision required on either side of the proportion (Sayed, 2007). The assumptions for sample size calculations were $95 \%$ confidence interval, 50\% frequency of adherence, and 5\% worst acceptable result. Hence, $S$ became 384, but when the adjusted sample size was calculated, using $S /(1+S / P o p)$, it resulted in a sample size of 355 patients.

\section{Research Instrument}

Specifically designed structured interview schedules were used to collect data from the respondents. The interview schedule was piloted with 10 patients who were excluded from participating in the actual study. During the pilot phase, the interviewers identified ambiguous words and sentences. The interviewers helped the researchers to rephrase some items. At the conclusion of each interview, the patient was asked how s/he felt about the nature, style, and timing of the questions. All identified problems were addressed during revision of the data collection instrument. Individual questions were structured to avoid ambiguities, and interviewers were trained to ask questions in a uniform way.

The interviews were conducted in Amharic, the national language of Ethiopia, which was understood by all patients. A licensed translator translated the original interview schedule in English into Amharic. Data collectors were well versed in both English and Amharic. They were recruited based on college-level education and prior experience with data collection. Hence, the data collectors accurately documented the respondents' Amharic responses, which the first author then translated into English. Thereafter, an English-Amharic translator checked the data for accuracy.

The interview schedule was divided into components. Section A asked general and demographic questions, including background information about the respondents, such as the ART patients' ages, gender, residence types, levels of education, and marital status. Questions in Section B inquired how long 
each patient had known about his/her HIV infection, ART adherence, and ART regimen. Adherence levels were calculated by dividing medication doses taken by medication doses prescribed. Section $C$ assessed each patient's knowledge about HIV and ART. Questions focused on knowledge about HIV transmission, HIV prevention, and ART. Section D addressed stigma and discrimination experienced by each patient, focusing on the patients' experiences of stigma and discrimination. Section E pertained to symptoms of depression. The questions were asked in such a way that patients could respond to each question as either yes or no. The total number of yes responses was summed to reach a potential diagnosis of depression. Section $\mathrm{F}$ asked questions about alcohol use to gather data on the frequency of alcohol consumption.

\section{Data Collection Procedure}

The research proposal was accepted and ethical clearance was granted by the Research and Ethics Committee of the Department of Health Studies, University of South Africa, and by the participating hospital's institutional review board. This was done to assure that the rights and welfare of the individuals involved were protected, the appropriate methods were used to secure informed consent, and the potential benefits of the investigation were greater than the risks.

Nine patients were interviewed per day (during work days) for 2 months, from November 2010 to January 2011. Two data collectors conducted the structured interviews and the first author checked the completed interview schedules.

\section{Data Analysis}

Different statistics and measures of association were applied to test a null hypothesis at $p$-value $<.05$ and $95 \%$ confidence levels to calculate a range of likely values for the differences in mean outcomes (adherence levels) of the respondents. Because the sample size was larger than 60 , $z$-tests were used to calculate $p$-values for those dependent variables that were continuous and that were independent categorical variables. There were also exposure variables with more than two categories, and hence, analysis of variance calculations were conducted specifically to compare adherence levels between different cate- gories of alcohol users. The adherence percentage was calculated by dividing the number of pills taken by the number of pills prescribed, for a specific number of days. The Statistical Package for the Social Sciences (SPSS version 17.0; IBM, Armonk, NY) computer software was used.

\section{Results}

\section{Patients' Levels of ART Adherence}

Of the 355 patients, $26.5 \%(n=94)$ were nonadherent, with less than $95 \%$ adherence levels; $73.5 \%(n=$ 261) were adherent at $95 \%$ to $100 \%$ adherence levels; only $1.7 \%(n=6)$ of the patients had $0 \%$ to $5 \%$ adherence levels. Adherence levels ranged from $0 \%$ to $100 \% ; 0 \%$ indicated that the patient did not take any pills during the preceding 7 days and $100 \%$ indicated that the patient took all prescribed pills during the previous 7 days. As many as $73.5 \%(n=261)$ of the respondents took their ARVs as prescribed.

\section{Gender versus ART Adherence}

Of the 94 nonadherent respondents, $43.6 \%(n=$ $41)$ were male and $56.4 \%(n=53)$ were female. Among the 261 adherent patients, $34.1 \%(n=89)$ were male. The adherence level of males was significantly different from that of females $(p=.05,95 \%$ confidence interval $[\mathrm{CI}]=-6.40$ to 0.01 ), with females being more adherent than males.

\section{Age versus ART Adherence}

Patients' ages ranged from 20 to 67 years, with an average age of 36.4 years. The 30- to 34-year-old age group constituted the largest group of patients $(n=$ 77), followed by the 35- to 39-year-old age group $(n=72)$. The most adherent patients were identified in the age group 30 to 34 years $(n=55)$, followed by the 35- to 39-year-old age group $(n=53)$. In the 25to 29-year-old age group, there were 47 adherent respondents. Age was not significantly associated with adherence $(p=.15)$.

\section{Marital Status versus ART Adherence}

Of 261 adherent patients, 115 (44.1\%) were married and $67(25.7 \%)$ had been widowed. There 
was no association between marital status and adherence levels ( $p=.30$, two-tailed $\chi^{2}$ test).

\section{World Health Organization HIV Stage versus ART Adherence Levels}

Of 355 respondents, 179 (50.4\%) were classified as being in WHO Stage I, $83(23.4 \%)$ in Stage II, $57(16.1 \%)$ in Stage III, and $14(3.9 \%)$ in Stage IV. The rest, $22(6.2 \%)$, did not have WHO staging reported in their records. Most of the ART-adherent patients were found in WHO Stage I $(57.8 \% ; n=$ $141)$, followed by WHO Stage II $(23.0 \% ; n=56)$. There was no significant association between WHO staging and adherence $(p=.039)$, implying that the proportion of patients who adhered to treatment did not increase as the WHO clinical stage decreased.

\section{Correlation of Adherence versus Knowledge Levels}

The mean knowledge score for our sample was $61.84 \%$ (range $18.89 \%-86.66 \%$; SD 10.92). Spearman's Correlation Coefficient was -0.002 , suggesting no association between adherence level and knowledge score. At the 5\% significance level, the results also indicated no statistically significant association $(p=.967)$. People with higher knowledge scores (good knowledge of HIV and ART) were not found to be more adherent to ART than those with poorer knowledge levels as measured in this study.

\section{Adherence versus Internal Stigma}

Of the 261 adherent patients, 161 (61.7\%) did not develop self-stigma. Adherence levels ranged from $0 \%$ to $100 \%$ for patients experiencing both internalized stigma and noninternalized stigma. At a 5\% significance level, the $z$-test statistic showed a significant association between internalized stigma and adherence ( $p$ $=.003 ; 95 \% \mathrm{CI}-7.70$ to -1.55$)$. This finding indicated that people with internalized stigma were less likely to adhere to prescribed ART. Internal stigma was associated with ART adherence for both males and females (OR for male $=2.9,95 \%$ CI 1.4 to 6.3; $\chi^{2}=.005$; OR for females $=3.1,95 \%$ CI 1.6 to 5.8 ; $\left.\chi^{2}=.005\right)$, but no statistically significant genderrelated differences could be identified. This implied that internal stigma negatively affected ART adherence levels for both men and women because men experiencing internal stigma were 2.9 times more likely and women experiencing internal stigma were 3.1 times more likely to be nonadherent to ART than those who did not experience internal stigma.

\section{Adherence versus Perceived Stigma}

Of the 94 nonadherent patients, 34 (36.2\%) had perceived stigma, and of the 261 adherent patients, $26(10.0 \%)$ reported experiencing perceived stigma. Adherence levels ranged from $0 \%$ to $100 \%$ in both groups (those who developed perceived stigma and those who did not). However, of 6 patients with an adherence level of $0 \%$ to $5 \%, 2(33 \%)$ developed perceived stigma and $4(67 \%)$ did not. The $z$-test showed a statistically significant association at a 5\% level between perceived stigma and adherence $(p=.01 ; 95 \%$ CI -11.66 to -3.54$)$ for men and women combined. This finding indicated that ART adherence levels tended to be lower if patients experienced perceived stigma. Women's ART adherence levels (OR 6.9; 95\% CI 3.3 to $14.6 ; \chi^{2}=0.0001$ ) seemed to be slightly more affected by perceived stigma than those of men (OR 3.3; 95\% CI 1.3 to 8.6; $\chi^{2}=0.014$; Figure 1).

\section{Adherence versus Discrimination}

Of the 62 patients experiencing perceived discrimination, $24(38.7 \%)$ were nonadherent, and of the 293 patients who did not experience discrimination, 70 $(23.9 \%)$ were nonadherent. Adherence levels ranged from $0 \%$ to $100 \%$ for both groups of patients (experiencing vs. not experiencing discrimination). Of the 261 patients with adherence levels of $95 \%$ to $100 \%$, $38(14.6 \%)$ faced discrimination and $223(85.4 \%)$ did not encounter discrimination. The $z$-test showed a statistically significant association between discrimination and adherence at the 5\% significance level $(p=$ $.007 ; 95 \%$ CI -9.6 to -1.6 ). Patients who experienced discrimination had lower levels of adherence.

A gender-based statistical analysis indicated that women's ART adherence levels (OR 2.2; 95\% CI 1.1 to $\left.4.5 ; \chi^{2}=4.983\right)$ were more adversely affected by discrimination than those of men (OR 1.9; 95\% CI 0.7 to $\left.5.3 ; \chi^{2}=1.612\right)$, as indicated in Table 1 . Women who endured discrimination were 2.2 (OR 


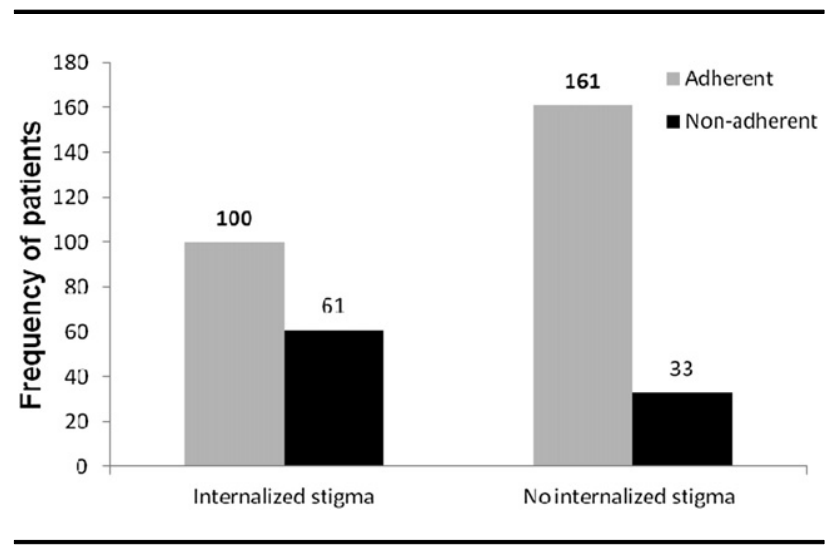

Figure 1. Adherence level versus internal stigma $(n=355)$.

2.2) times more likely to be nonadherent to ART than those women who did not experience discrimination.

\section{Adherence versus Depression}

Of the 355 respondents, 19 (5.4\%) developed depression. Among the 19 depressed patients, 15 $(78.9 \%)$ were nonadherent and $4(21.1 \%)$ were adherent. Six $(31.6 \%)$ of the depressed patients had an adherence level of $85 \%$ to $89 \% ; 257(76.5 \%)$ of the patients who did not suffer from depression had an adherence level of $95 \%$ to $100 \%$. There was a statistically significant association between adherence and depression at the 5\% level $(p=.046$; $95 \% \mathrm{CI}-13.81$ to -0.12 ; Figure 2).

\section{Adherence versus Alcohol Use}

Of the 94 nonadherent respondents, $40(42.6 \%)$ were infrequent light drinkers, $50(53.2 \%)$ were current abstainers, $1(1.1 \%)$ was a frequent light drinker, and 3 (3.2\%) were infrequent heavy drinkers. There was a statistically significant association between alcohol use and adherence at the 5\% significance level $(p=.001)$. This indicated that when the frequency and amount of alcohol consumption was high, the probability of ART nonadherence was also high.

\section{Discussion}

Our study reported that $261(73.5 \%)$ patients were ART adherent at a $95 \%$ to $100 \%$ level. These support the findings of other studies that found that patients in resource-poor countries could adhere to prescribed ARVs and derive benefits from ART (Carlucci et al., 2008; Grant et al., 2008). We also found that age was not significantly associated with adherence $(p=.15)$. Similar results were reported in a Nigerian study showing no significant association between age and ART adherence levels (Afolabi, Ijadunola, Fatusi, \& Olasode, 2009).

Our study found no association between marital status and adherence levels ( $p=.30$, two-tailed $\chi^{2}$ test), but more women than men adhered to prescribed ART regimens. This finding was supported by a South African study showing that women were more adherent than men, based on self-reported adherence rates validated by viral suppression, CD4+T cell counts, and clinical outcomes (Boulle, Michaels, \& Hildebrand, 2004).

No correlation could be established in our study between patients' knowledge levels and their ART adherence levels. Other researchers (Grant et al.,

Table 1. Gender-Related Risk Estimate: Discrimination Experienced By Males and Females versus ART Adherence Level

\begin{tabular}{|c|c|c|c|c|c|}
\hline \multirow[b]{2}{*}{ Gender } & & & \multicolumn{2}{|c|}{ Adherence Level } & \multirow[b]{2}{*}{ Total } \\
\hline & & & $<95 \%$ & $\geq 95 \%$ & \\
\hline \multirow[t]{4}{*}{$\overline{\text { Male }}$} & Experienced discrimination & Discriminated against by other people & 8 & 10 & $\overline{18}$ \\
\hline & & Not discriminated against by other people & 33 & 79 & 112 \\
\hline & Total & & 41 & 89 & 130 \\
\hline & Male Pearson $\chi^{2} 1.612(d f=$ & totic significance [2-sided] $.204 ; p=.039$ ) & & & \\
\hline \multirow[t]{3}{*}{ Female } & Experienced discrimination & Discriminated against by other people & 16 & 28 & 44 \\
\hline & & Not discriminated against by other people & 37 & 144 & 181 \\
\hline & Total & & 53 & 172 & 225 \\
\hline
\end{tabular}

NOTE: Female Pearson $\chi=4.983(d f=1$; asymptotic significance [2-sided] .026; $p=.039)$; ART $=$ antiretroviral therapy. 


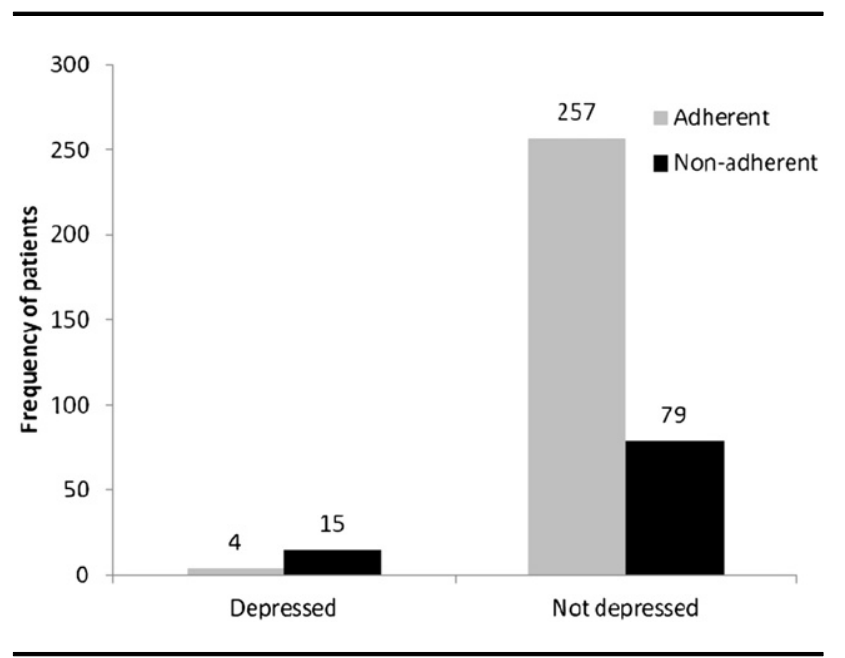

Figure 2. The relationship between adherence and depression $(n=355)$.

2008), however, determined that patients' enhanced knowledge levels could help to improve their ART adherence levels.

Respondents with advanced WHO clinical stages had lower levels of ART adherence than those in the initial WHO clinical stage, probably indicating that ART adherence improved the patients' clinical conditions or prevented deterioration of their clinical conditions, but this was not statistically significant. No evidence was found that improved clinical conditions of the patients in our study encouraged others to be adherent to their ART regimens, unlike the findings of a Zambian study (Grant et al., 2008).

People with internalized stigma were less likely to take their prescribed ARVs in our study, but no significant gender-related results were obtained. According to a study conducted in the United States, internal stigma and other factors such as denial, life stress, and adverse ARV side effects were barriers to ART adherence (Konkle-Parker, Erlen, \& Dubbert, 2008).

ART adherence tended to be lower in both male and female patients who encountered perceived stigma, according to our findings. Similar findings were reported in a study done in five other African countries (Lesotho, Malawi, South Africa, Swaziland, and Tanzania), which reported that perceived stigma interfered with adherence to ART and correlated with missed doses of ARVs (Dlamini et al., 2009).

Our respondents who experienced discrimination had lower levels of ART adherence. Women's ART adherence levels were more adversely affected by discrimination than those of men. This finding is supported by another study, which indicated that stigma and discrimination could contribute to PLWH not taking their medications at the correct time or in the correct way, in order to hide their HIV infection (Department for International Development, 2007). Our respondents did not report decreased discrimination as patients regained their health, unlike the findings of a Zambian study (Grant et al., 2008).

There was a statistically significant association between adherence and depression for our respondents. An Ethiopian study (Amberbir, Woldemichael, Getachew, Girma, \& Deribe, 2008) reported that nondepressed patients maintained double the ART adherence rates that depressed patients could manage to maintain.

There was a statistically significant association between alcohol use and adherence. Alcohol use was associated with ART nonadherence even though it could not be concluded that alcohol consumption caused low ART adherence rates (Hendershot, Stoner, Pantalone, \& Simoni, 2008).

\section{Recommendations}

Our study allowed us to conclude that revisions to clinical care could make a difference in ART adherence. If patients could be helped to address personal factors influencing their ART adherence levels, their $\mathrm{CD} 4+\mathrm{T}$ cell counts should increase and their viral loads should decrease, enabling them to enjoy higher levels of well-being and reducing their chances of developing ARV-resistant strains of HIV.

\section{Conclusion}

We studied 355 HIV-infected patients who were on ART in Addis Ababa, Ethiopia, to identify personal factors related to treatment adherence. These adherence level findings confirm that even in resourcelimited countries, patients can maintain high levels of ART adherence. To improve ART adherence and subsequent improved health outcomes, it is necessary to design and implement practically sound programs that alleviate the burdens of stigma and 
discrimination, depression, and alcohol use among ART patients.

\section{Limitations}

The findings of our study need to be interpreted within the context of the limitations of the study. These limitations include that ART adherence levels might be influenced by different personal factors among ART patients at other hospitals, private hospitals, and health care centers. Patients younger than 18 years of age, who had been on ART for less than 12 months, or who had discontinued their ART, might have encountered different personal challenges to adhere to their ART regimens. Consequently, the results of our study might not be generalizable beyond the ART patients who received their treatment at the clinic that was our study site. In addition, cultural issues were not addressed and might have influenced patients' ART adherence levels. In-depth interviews with individual patients might have revealed insight into patients' experiences of living with HIV and taking ARVs every single day for the rest of their lives.

\section{Clinical Considerations}

Antiretroviral therapy (ART) adherence could be enhanced if nurses and other health care workers would:

- Identify each patient's ART adherence at every clinic visit and investigate reasons for decreased ART adherence levels.

- Watch patients on ART for signs of depression and/or alcoholism; treat patients for these conditions or refer for appropriate treatment.

- Enable patients on ART to verbalize internal and external experiences of victimization and discrimination and help them to find meaningful ways of coping with these challenges.

- Address all the above issues before considering any ART regimen change in cases of immunological and/or virological failure.

\section{Disclosures}

The authors report no real or perceived vested interest that relate to this article that could be construed as a conflict of interest.

\section{Acknowledgments}

No funding was received for conducting this study. The authors thank the authorities for granting permission to conduct the study and the respondents who agreed to be interviewed.

\section{References}

Afolabi, M. O., Ijadunola, K. T., Fatusi, A. O., \& Olasode, O. A. (2009). Determinants of adherence to antiretroviral drugs among people living with HIV/AIDS in the Ife-Ijesa zone of Osun state, Nigeria. African Journal of Primary Health Care \& Family Medicine, 1(1), 6-1 http://dx.doi.org/10. 4102/phcfm.v1i1.6

Amberbir, A., Woldemichael, K., Getachew, S., Girma, B., \& Deribe, K. (2008). Predictors of adherence to antiretroviral therapy among HIV-infected persons: A prospective study in Southwest Ethiopia. Retrieved from http://www.ncbi.nlm. nih.gov/pmc/articles/PMC2518153/?tool=pmcentrez

Boulle, A., Michaels, D., \& Hildebrand, K. (2004). Gender aspects of access to ART and treatment outcomes in a South African township. Retrieved from http://gateway.nlm.nih.gov/ MeetingAbstracts/ma?f $=102279276 . \mathrm{html}$

Burns, N., \& Grove, S. K. (2005). The practice of nursing research: Conduct, critique, and utilization ( $5^{\text {th }}$ ed.). St. Louis, MO: Elsevier-Saunders.

Carlucci, J. G., Kamanga, A., Sheneberger, R., Shephered, B. E., Jenkins, C. A., Spurrier, J., \& Vermund, S. H. (2008). Predictors of adherence to antiretroviral therapy in rural Zambia. Journal of Acquired Immune Deficiency Syndrome, 47(5), 615-622.

Department for International Development. (2007). Taking action against HIV stigma and discrimination. Retrieved from http://www.aidslex.org/site_documents/D-0024E.pdf

Dlamini, P. S., Wantland, D., Makoae, L. N., Chirwa, M., Kohi, T. W., Greeff, M., \& Holzemer, W. L. (2009). HIV stigma and missed medications in HIV-positive people in five African countries. AIDS Patient Care and STDS, 23(5), 377-387.

EngenderHealth. (2004). Reducing stigma and discrimination related to HIV and AIDS. Training for health care workers. New York, NY: Printech.

Ethiopian Federal Ministry of Health HIV/AIDS Prevention and Control Office. (2006). AIDS in Ethiopia. Sixth report. 
Retrieved from http://www.etharc.org/aidsineth/publications/ singlepointprev_2006

Ethiopian Federal Ministry of Health HIV/AIDS Prevention and Control Office. (2007). Single point HIV prevalence estimate. Retrieved from http://www.etharc.org/aidsineth/publications/ singlepointprev_2007

Ethiopian Federal Ministry of Health HIV/AIDS Prevention and Control Office. (2009). Monthly HIV care and ART update. Retrieved from http://www.etharc.org

Grant, E., Logie, D., Masura, M., Gorman, D., \& Murray, S. A. (2008). Factors facilitating and challenging access and adherence to antiretroviral therapy in a township in the Zambian Copperbelt. AIDS Care, 20(10), 1155-1160.

Hendershot, C. S., Stoner, S. A., Pantalone, D. W., \& Simoni, J. M. (2008). Alcohol use and anti-retroviral adherence: Review and meta-analysis. Retrieved from http://www.ncbi. nlm.nih.gov/pmc/articles/PMC2815237/?report=abstract \& tool $=$ pmcentrez

Konkle-Parker, D. J., Erlen, J. A., \& Dubbert, P. M. (2008). Barriers and facilitators to medication in a southern minority population with HIV disease. Journal of the Association of Nurses in AIDS Care, 19(2), 98-104. http://dx.doi.org/10. 1016/j.jana.2007.09.005

Markos, E., Worku, A., \& Davey, G. (2008). Adherence to ART in PLWHA at Yirgalem Hospital, South Ethiopia. The Ethiopian Journal of Health Development, 22(2), 174-179.

Reus, V. I. (2005). Mental disorders. In D. L. Kasper, A. S. Faucy, D. L. Longo, E. Braunwald, S. L. Hausler, \& S. L. Jameson (Eds.), Harrison's principles of internal medicine $\left(16^{\text {th }}\right.$ ed.) (pp. 2547-2561). New York, NY: McGrawHill.

Sayed, A. R. (2007). Sample size calculation. In G. Joubert, \& R. Ehrlich (Eds.), Epidemiology. A research manual for South Africa (pp. 346-348). Cape Town, South Africa: ABC Press.

World Health Organization. (2003). Adherence to long term therapies: Evidence for action. Retrieved from www.who.int/chp/ knowledge/publications/adherence_report/en]

World Health Organization. (2006). Strengthening health services to fight HIV/AIDS. Antiretroviral therapy for HIV infection in adults and adolescents: Recommendation for a public health approach. Retrieved from http://www.who. int/hiv/pub/arv/adult/en

Wutoh, A. K., Brown, C. M., Kumoji, E. K., Daftary, C. M., Jones, T., Barness, N. A., \& Powell, N. J. (2001). Antiretroviral adherence and use of alternative therapies among older HIV-infected adults. Journal of the National Medical Association of America, 93(7), 243-250. 